\title{
Secure exchange of information by synchronization of neural networks
}

\author{
Ido Kanter ${ }^{1}$, Wolfgang Kinzel ${ }^{2}$ and Eran Kanter ${ }^{1}$
}

(1) Department of Physics, Bar Ilan University, 52900 Ramat Gan, Israel (2) Institut für Theoretische Physik, Universität Würzburg, Am Hubland, D-97074 Würzburg, Germany

3. 9. 2001

\begin{abstract}
A connection between the theory of neural networks and cryptography is presented. A new phenomenon, namely synchronization of neural networks is leading to a new method of exchange of secret messages. Numerical simulations show that two artificial networks being trained by Hebbian learning rule on their mutual outputs develop an antiparallel state of their synaptic weights. The synchronized weights are used to construct an ephemeral key exchange protocol for a secure transmission of secret data. It is shown that an opponent who knows the protocol and all details of any transmission of the data has no chance to decrypt the secret message, since tracking the weights is a hard problem compared to synchronization. The complexity of the generation of the secure channel is linear with the size of the network.
\end{abstract}

PACS numbers: 87.18.Sn,89.70.+c

The ability to build a secure channel is one of the most challenging fields of research in modern communication. Since the secure channel has many applications, in particular for mobile phone, satellite and internet-based communications, there is a need for fast, effective and secure transmission protocols [1]. Here we present a novel principle of a cryptosystem based on a new phenomenon which we observe for artificial neural networks.

The goal of cryptography is to enable two partners to communicate over an insecure channel in such a way that an opponent cannot understand and 
decrypt the transmitted message. In a general scenario, the message is encrypted by the sender through a key $E_{k}$ and the result, the ciphertext, is sent over the channel. A third party, eavesdropping on the channel, should not determine what the message was. However, the recipient who knows the encryption key can decrypt the ciphertext using his private key $D_{k}$.

In a private-key system the recipient has to agree with the sender on a secret key $E_{k}$, which requires a hidden communication prior to the transmission of any message. In a public-key system, on the other side, the key $E_{k}$ is published and a hidden communication is not necessary. Nevertheless, an opponent cannot decrypt the transmitted message since it is computationally infeasible to invert the encryption function without knowing the key $D_{k}$. In a key-exchange protocol, both partner start with private keys and transmit - using a public protocol - their encrypted private keys which, after some transformations, leads to a common secret key. In most applications a publickey system is used which is based on number theory where the keys are long integers [1, 2].

In this report we suggest a novel cryptosystem. It is a key-exchange protocol which does neither use number theory nor a public key, but it is based on a learning process of neural networks: The two participants start from a secret set of vectors $E_{k}(0)$ and $D_{k}(0)$ without knowing the key of their partner. By exchanging public information the two keys develop to a common time dependent key $E_{k}(t)=-D_{k}(t)$, which is used to encrypt and decrypt a given message. An opponent who knows the algorithm and observes any exchange of information is not able to find the keys $E_{k}(t)$ and $D_{k}(t)$. Our method is based on a new phenomenon presented here: Synchronization of neural networks by mutual learning [3].

Simple models of neural networks describe a wide variety of phenomena in neurobiology and information theory [4, 5, 6. Artificial neural networks are systems of elements interacting by adaptive couplings which are trained from a set of examples. After training they function as content addressable associative memory, as classifiers or as prediction algorithms.

In this report we present a new phenomenon: Two feedforward networks can synchronize their synaptic weights by exchanging and learning their mutual outputs for given common inputs. Surprisingly, synchronization is fast; the number of bits required to achieve perfect alignment of the weights is lower than the number of components of the weights. After synchronization, the synaptic weights define the common time dependent private key $E_{k}(t)=-D_{k}(t)$. With respect to possible applications we find that first, 
tracking the weights of one of the networks by the opponent is a hard problem. Although we were not able to find a mathematical proof, our simulations, in addition to arguments based on analytic results on neural networks, give clear evidence that our key exchange protocol is secure [18]. Second, the complexity of our cryptosystems scales linearly with the size of the network (=number of bits of the keys). In summary, from this new biological mechanism one can construct efficient encryption systems using keys which change permanently.

This phenomenon, as well as the corresponding applications in cryptography, can be extended to a system of several partners communicating with each other, as well as to other tasks relying on a secure channel [18]. Since synchronization is a subject of recent research in neuroscience too [7, 8, 9], we believe that our bridge between the theory of neural networks and cryptography may help to understand communication between parts of biological neuronal or genetic networks.

In the following we introduce and investigate a simple model which shows the properties sketched above. The architecture used by the recipient and the sender is a two-layered perceptron, exemplified here by a parity machine (PM) with $K$ hidden units. More precisely, the size of the input is $K N$ and its components are denoted by $x_{k j}, k=1,2, \ldots, K$ and $j=1, \ldots, N$. For simplicity, each input unit takes binary values, $x_{k j}= \pm 1$. The $K$ binary hidden units are denoted by $y_{1}, y_{2}, \ldots, y_{K}$. Our architecture is characterized by non-overlapping receptive fields (a tree), where the weight from the jth input unit to the kth hidden unit is denoted by $w_{k j}$, and the output bit $O$ is the product of the state of the hidden units (see Fig. 1). For simplicity we discuss PMs with three hidden units $K=3$. We use integer weights bounded by $L$, i.e. $w_{k j}$ can take the values $-L,-L+1, \ldots, L$.

The secrete information of each of the two partners are the initial values for the weights, $w_{k j}^{S}$ and $w_{k j}^{R}$, for the sender and the recipient, respectively. It consists of $6 N$ integer numbers, $3 N$ of the recipient and $3 N$ of the sender. Sender and recipient do not know the initial numbers of their partners, which are used to construct the common secret key.

Now each network is trained with the output of its partner. At each training step, for the synchronization as well as for the encryption/decryption step, a new common public input vector $\left(x_{k j}\right)$ is needed for both the sender and the recipient. For a given input, the output is calculated in the following two steps. In the first one, the state of the three hidden units, $y_{k}^{S / R}, k=$ $1,2,3$, of the sender and the recipient are determined from the corresponding 


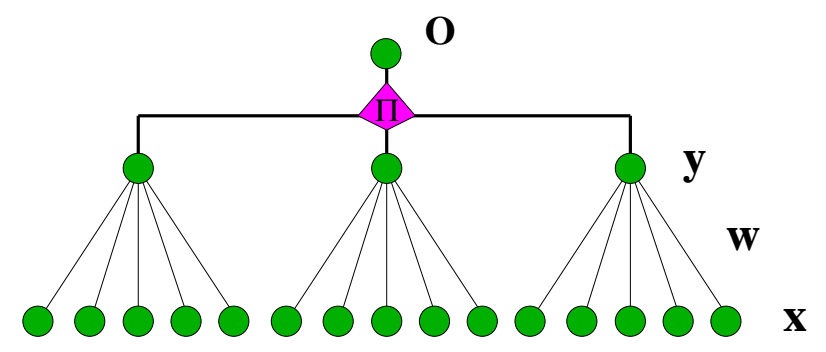

Figure 1: Architecture of the networks: $3 N$ input units $x$ are transmitted by three weight vectors $w$ to three hidden units $y$. The final output bit $O$ is the product of the hidden units.

fields

$$
y_{k}^{S / R}=\operatorname{sign}\left[\sum_{j=1}^{N} w_{k j}^{S / R} x_{k j}\right]
$$

In the case of zero field, $\sum w_{k j}^{S / R} x_{k j}=0$, the sender/recipient sets the hidden unit to $1 /-1$. In the next step the output $O^{S / R}$ is determined by the product of the hidden units, $O^{S / R}=y_{1}^{S / R} y_{2}^{S / R} y_{3}^{S / R}$.

The sender is sending its output (one bit) to the recipient, the recipient is sending its output to the sender and both networks are trained with the output of its partner. In case that they do not agree on the current output, $O^{S} O^{R}<0$, the weights of the sender/recipient are updated according to the following Hebbian learning rule [6, 10].

$$
\begin{array}{rll}
\text { if }\left(O^{S / R} y_{k}^{S / R}>0\right) & \text { then } & w_{k j}^{S / R}=w_{k j}^{S / R}-O^{S / R} x_{k j} \\
\text { if }\left(\left|w_{k j}^{S / R}\right|>L\right) & \text { then } & w_{k j}^{S / R}=\operatorname{sign}\left(w_{k j}^{S / R}\right) L
\end{array}
$$

Only weights belonging to the one (or three) hidden units which are in the same state as that of their output unit are updated, in each one of the two networks. Note that by using this dynamical rule, the sender is trying to imitate the response of the recipient and the recipient is trying to imitate the one of the sender.

There are three main ingredients in our model which are essential for a secure key exchange protocol: First, from the knowledge of the output, the internal representation of the hidden units is not uniquely determined because there is a four fold degeneracy (for the output +1 there are four internal representations for the three hidden units $(1,1,1),(1,-1,-1),(-1,1,-1),(-1,-1,1))$. 
As a consequence, an observer cannot know which of the weight vectors is updated according to equation (2). Second, we have chosen the parity machine since in the case of static weights, it is known that an opponent cannot obtain any knowledge about the rule if he is trained with less than $\alpha(L) N$ random examples (where $\alpha(L)=2.63$ for $L=3$ and for more details see [11]). This analytic result favours the PM over other multilayer networks. Third, since each component is bounded by $L$, an observer cannot invert the sum of equation (2); the network forgets [12]. As a consequence of these three ingredients, the initial weight vectors cannot be recovered from the knowledge of the time dependent synchronized keys. All three of these mechanisms hidden units, PM as well as bounded weights - make the problem hard for any observer.

We find that the two PMs learning from each other are able to synchronize, at least for some parameters $K, L$ and $N$ [13]. Our simulations show that after an initial relatively short transient time the two partners align themselves into antiparallel states. It is easy to verify from our learning rule that as soon as the two networks are synchronized they stay so forever. The number of time steps to reach this state depends on the initial weight vectors and on the sequence of random inputs, hence it is distributed. Fig. 2 shows the distribution of synchronization time obtained from at least 1000 samples. It is evident that two communicating networks synchronize in a rather short time. The average synchronization time $t_{a v}$ decreases with increasing size $N$ of the system, see Fig. [3; it seems to converge to $t_{a v} \simeq 410$ for infinitely large networks. Surprisingly, in the limit of large $N$ one needs to exchange only about 400 bits to obtain agreement between $3 N$ components. However, one should keep in mind that the two partners do not learn the initial weights of each other, they just are attracted to a dynamical state with opposite weight vectors.

As soon as the weights of the sender and the recipient are antiparallel the public initialization of our private-key cryptosystem is terminated successfully and the encryption of the message starts. Now there are two possibilities to choose an algorithm: First, use a conventional encryption algorithm, for example a stream cipher like the well-known Blum-Blum-Shub bit generator [1]. In this case the seed for this pseudo-random number generator is constructed from our weight vector after synchronization. Second, use the PM itself for a stream cipher by multiplying its output bit with the corresponding bit of the secret data.

In the case of the PM, the complexity of the encryption/decryption pro- 


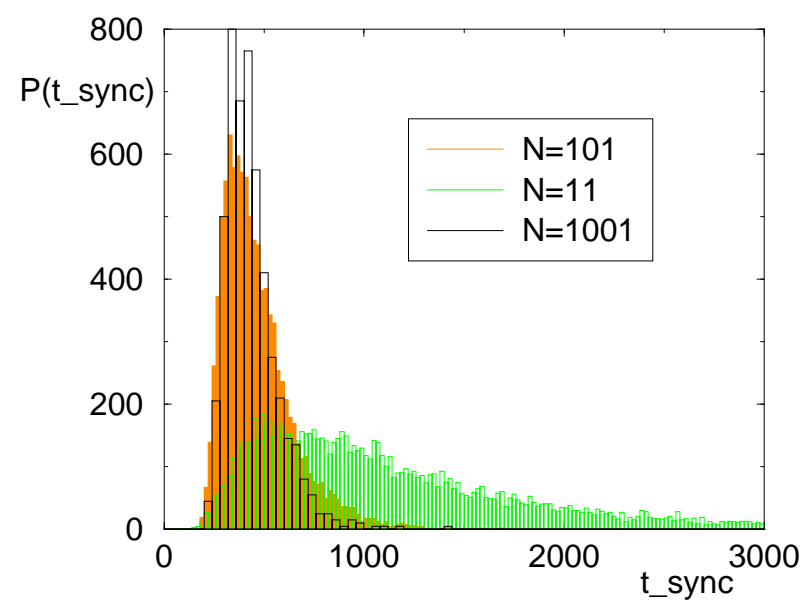

Figure 2: Distribution of synchronization time $t_{\text {sync }}$ for three sizes $N$ of the two networks.

cesses scales linearly with the size of the transmitted message, whereas the complexity of the synchronization process does not scale with the size of the network. Hence our construction is a linear cryptosystem [14].

Now we examine a possible attack on our cryptosystem. The opponent eavesdropping on the line knows the algorithm as well as the actual mutual outputs, hence he knows in which time steps the weights are changed. In addition, the opponent knows the input $x_{k j}$ as well. However, the opponent does not know the initial conditions of the weights of the sender and the recipient. As a consequence, even for the synchronized state, the internal representations of the hidden units of the sender and the recipient are hidden from the opponent and he does not know which are the weights participating in the learning step. For random inputs all four internal representations appear with equal probability in any stage of the dynamical process, hence for $t$ training steps there are $4^{t}$ possibilities to select internal representations.

Therefore, on the time scale of synchronization the observing network has no chance to obtain complete knowledge about the other two networks. We have simulated attacks of an observer, assuming that the most effective algorithm is a network which has identical architecture to the recipient: A PM with the same learning rule and parameters as described above. The observing network is trained with the input vector and output bit of the sender, and the training step (2) is performed only if sender and recipient 


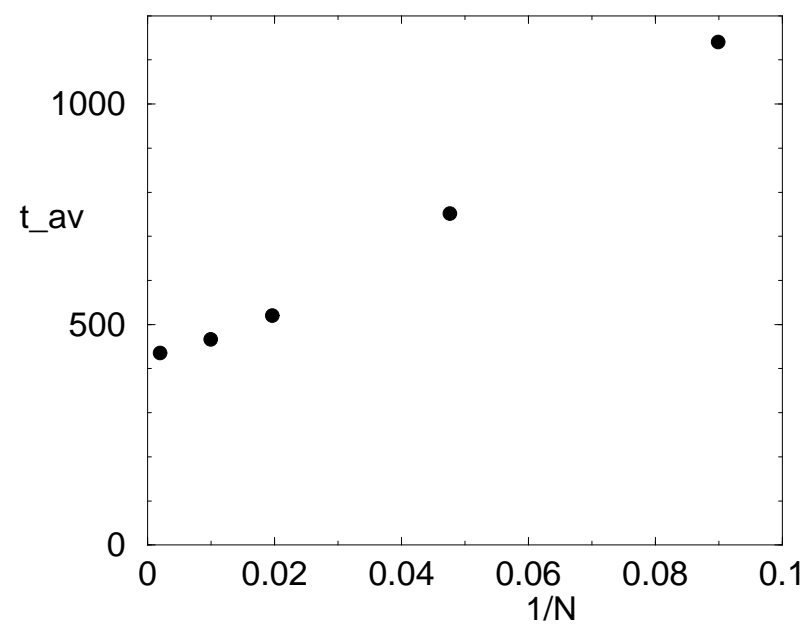

Figure 3: Average synchronization time as a function of $1 / N$, for system size $N=11,21,51,101,1001$.

disagree with each other. Note that the sender does not react to the output of the opponent, which results in a large noise to signal ratio compared with the recipient 18.

The learning rule (2) may be considered for each component of the weight vectors as a kind of biased random walk with reflecting boundaries. Therefore, for very long times, the observer may take the weight vector of the other network by chance. The distribution of the ratio between the time $t_{\text {sync }}$ the sender and recipient need to synchronize and the learning time $t_{\text {learn }}$ the opponent needs for complete overlap is shown in Fig. 4 . For $N=101$ the average learning time is a factor of about 125 larger than the corresponding synchronization time. In addition, with increasing system size the tail of the distribution for larger ratios is reduced.

Hence the time to synchronize by chance is very long and in the example discussed here it is of order $O\left(10^{5}\right)$ [16]. The heart of our cryptosystem is that synchronization is a much simpler task than tracking by an observer.

This principle is also supported by the following observation. Assume that the observer has already some knowledge about one of the networks, he knows $M$ out of $N$ components for each of the three hidden units. We have measured whether the observer succeeds to synchronize within 2000 time steps - a time much longer than the average synchronization time for the two communicating networks. For $M=N$ the observer is parallel to 


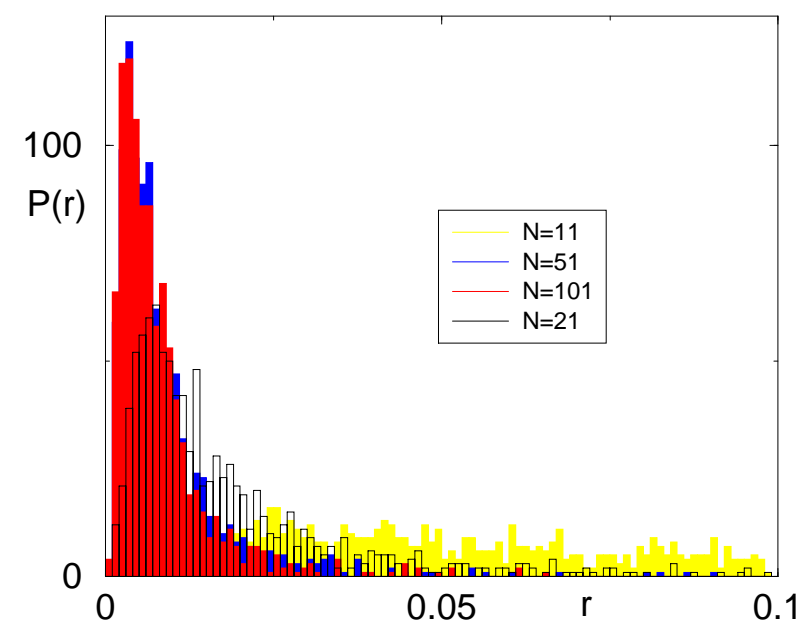

Figure 4: Frequency of the ratio $r$ between synchronization and learning times.

one of the networks and remains so forever. But already for $M=N-1$ we find a high probability that the weight vector of the observer separates completely from its almost parallel alignment. For smaller values of $M$ this probability decreases fast to zero. Surprisingly, even in case the observer has almost complete knowledge about the two partners, he does not succeed to achieve complete information from learning examples. This fact reduces the probability of the opponent to imitate one of the communicating networks using an ensemble of PMs.

Our key exchange protocol can be generalized to include Bit-Packages as is briefly described below. An important issue for the implementation of our cryptosystem is to accelerate the synchronization process from hundreds of time steps to a few dozens while keeping the security of our channel. Surprisingly, both of these two goals can be achieved simultaneously sending bit packages $(\mathrm{BP})$. In this scenario the process contains the following steps: (a) The sender and the recipient generate $B>1$ common inputs. (b) The sender and the recipient calculate the output of their PM for the set of $B$ inputs and store the $B$ sets of the corresponding values $y_{k i}(i=1, \ldots, B)$ of the hidden units (the internal representations) (c) The transmission of mutual information; the sender/recipient sends a package consisting of $B$ bits $\left(b_{i}^{S / R}\right)$ to the recipient/sender. (d) The sender and the recipient are updating their weights using the same learning rule as for $B=1$ : In case that bit $b_{i}^{S} \neq b_{i}^{R}$ 


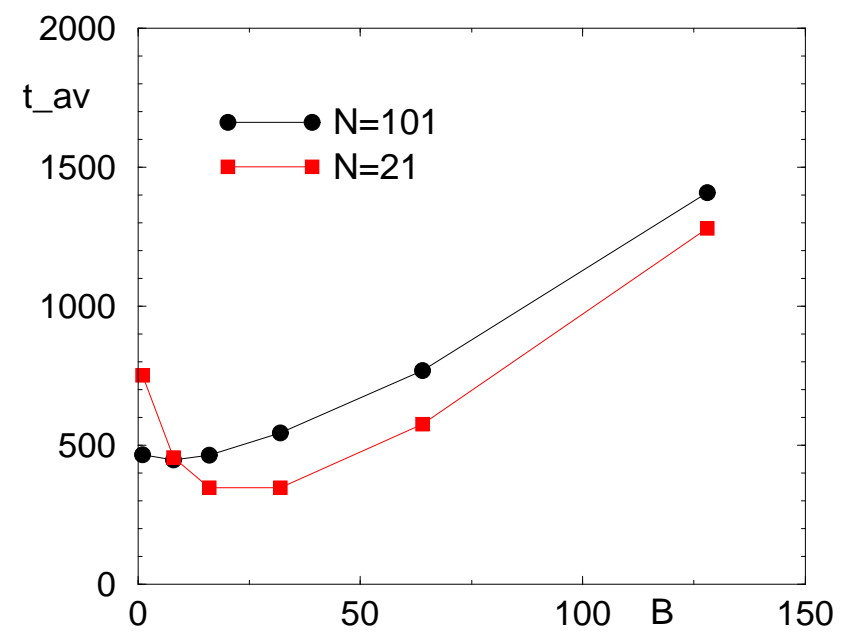

Figure 5: Total number of transmitted bits until synchronization. $B$ is the number of bits in each bit package exchanged between sender and recipient.

the learning process is taking place as before using the corresponding internal representations. The synchronization time is dramatically reduced, as is shown in Fig. 5. For instance, for $N=21, K=3, L=3$, synchronization is achieved after 12 bit packages if the size of the package is larger than $B \geq 32$.

Other extensions of our method as well as the analytical calculation of the distribution of $t_{\text {sync }}, t_{\text {learn }}$ for various $K, L$ and continuous weights, and version space of the PMs which are consistent with the training set will be dicussed in [18].

Finally, we want to remark that synchronization is a subject of recent research in neuroscience, where for instance, in experiments on cats and monkeys one has found that the spike activity of neurons in the visual cortex has correlations which depend on the kind of optical stimulus shown to the animal [17]. The phenomenon described here suggests that synchronization can be used by biological neuronal networks or by networks of the immune system to exchange secure information between different parts of an organism.

\section{References}


[1] D. R. Stinson, Cryptography: Theory and Practice (CRC Press 1995)

[2] R. L. Rivest, A. Shamir and L. Adelman, Comm. of the ACM 21, 120 (1978).

[3] Note that our method is based on a different principle than synchronization of chaotic maps, H. Strogatz, Nonlinear Dynamics and Chaos (Addison-Wesley, 1994).

[4] J. Hertz, A. Krogh, and R. G. Palmer: Introduction to the Theory of Neural Computation, (Addison Wesley, Redwood City, 1991)

[5] A. Engel, and C. Van den Broeck: Statistical Mechanics of Learning, (Cambridge University Press, 2001)

[6] M. Opper and W. Kinzel: Statistical Mechanics of Generalization, Models of Neural Networks III, ed. by E. Domany and J.L. van Hemmen and K. Schulten, 151-20 (Springer Verlag Heidelberg 1995)

[7] M. Castelo-Branco, R. Goebel, S. Neuensehwander and W. Singer, Nature 405, 685 (2000).

[8] J. K. Lin, U. Ernst and T. J.Sejnowski, Networks: Computation in Neural Systems 9, 333 (1998).

[9] A. Riehle, S. Grun, M. Diesmann and A. Aertsen, Science 278, 1950 (1997).

[10] M. Biehl and N. Caticha: Statistical Mechanics of On-line Learning and Generalization, The Handbook of Brain Theory and Neural Networks, ed. by M. A. Arbib (MIT Press, Berlin 2001)

[11] E. Barkai and I. Kanter, Europhys. Lett. 17, 181 (1991).

[12] J. L. van Hemmen, G. Keller and R. Kühn, Europhys. Lett. 5, 663 (1988).

[13] In fact, such a scenario has recently been studied by R. Metzler and W. Kinzel and I. Kanter, Phys. Rev. E 62, 2555 (2000) for two perceptrons with continuous weights and normalized weight vectors. However, we find that for such a system an outside observer can find the internal state of both perceptrons by learning the communication. Furthermore, the 
normalization of the weight vector after each learning step is a non-local operation and is violating the linear complexity of our cryptosystem.

[14] As for the hardware implementation of our cryptosystem, the calculation of the local fields, eq. (1), are similar to a linear filter which can be implemented in parallel using existing technologies. Hence, the complexities of the synchronization process is of $O(1)$ only.

[15] Another possible solution is based on continuous weights. A similar synchronization was observed for a PM with continuous weights, where the norm of the weights is set equal to 1 after each learning step [13, 18].

[16] Note that the average learning time for $N=101$ scales exponentially with $L$ and for $L=1,2,3,4$, was found in simulations to be equal to $58,1480,90380, \sim 10^{7}$.

[17] C. M. Gray, P. König, A. K. Engel and W. Singer, Nature 338, 334 (1989).

[18] I. Kanter, W. Kinzel and M. Rosenzvi (unpublished). 